\title{
The Infiniti Plus ultrasound needle guidance system improves needle visualization during the placement of spinal anesthesia
}

\author{
Hesham Elsharkawy ${ }^{1}$, Rovnat Babazade ${ }^{2}$, Sree Kolli ${ }^{3}$, Hari Kalagara ${ }^{3}$, and \\ Mounir L. Soliman ${ }^{3}$ \\ Departments of ${ }^{1}$ General Anesthesiology and Outcomes Research, CCLCM of Case Western Reserve University \\ Cleveland Clinic, ${ }^{2}$ Anesthesiology, University of Texas Medical Branch, Galveston, TX, and Outcomes Research \\ Consortium, Cleveland, OH, ${ }^{3}$ General Anesthesiology, Cleveland Clinic, Cleveland, OH, USA
}

Ultrasound guidance for neuraxial anesthesia has gained popularity over the last two decades [1]. This new application of technology also facilitates spinal anesthesia in patients with challenging anatomy, such as those with scoliosis or obesity, or elderly patients [2,3]. Infiniti Plus ${ }^{\mathrm{TM}}$ (CIVCO Medical Solutions, Kalona, IA, USA) is a needle-guidance system that was recently developed to assist clinicians in performing ultrasound-guided nerve blocks. A needle-guidance system allows efficient needle movement with improved needle shaft and tip visualization. A needle-guidance system has been used for peripheral nerve blocks with a high-frequency ultrasound probe [4]. We used the Infiniti Plus needle-guidance system with a low-frequency ultrasound probe in spinal anesthesia administered by a single physician to two patients with difficult spinal anesthesia.

A 62-year-old man was scheduled for left total hip replacement. He weighed $96 \mathrm{~kg}$ and had a body mass index of $30.4 \mathrm{~kg} / \mathrm{m}^{2}$. The L3-L5 spinous processes were not palpable, and the precise

Corresponding author: Hesham Elsharkawy, M.D., M.Sc.

Department of General Anesthesiology and Outcomes Research, CCLCM of Case Western Reserve University, 9500 Euclid Ave, E-30, Cleveland, OH 44195, USA

Tel: 1-216-445-3783, Fax: 1-216-444-2294

E-mail: elsharh@ccf.org

This study has been presented at The 38th Annual Regional Anesthesiology and Acute Pain Medicine Meeting, May 2013, Boston, Massachusetts, USA.

Received: November 24, 2015.

Accepted: December 2, 2015.

Korean J Anesthesiol 2016 August 69(4): 417-419

http://dx.doi.org/10.4097/kjae.2016.69.4.417 neuraxial midline was difficult to determine clinically. After obtaining informed consent, standard American Society of Anesthesiologists monitors and intravenous access was established and premedication with midazolam $2 \mathrm{mg}$ and fentanyl $50 \mu \mathrm{g}$ was given. He was positioned in the sitting position. Three trials of subarachnoid access without ultrasound guidance by an experienced anesthesiologist failed: bone was encountered in two trials, while in the third trial the cerebrospinal fluid (CSF) flow was not consistent (slow flow initially and no CSF was returned in the other three quadrants after turning the needle). Subsequently, spinal anesthesia was successful with an ultrasoundguided needle-guidance system at the first attempt.

The second case was a 62-year-old man who was scheduled for total hip replacement. Spinal anesthesia had been unsuccessful in the past. His medical history included kyphoscoliosis. No lumbar X-ray was available for a more detailed anatomical examination. On palpation, it was difficult to determine the midline. He initially refused spinal anesthesia, as his previous experience was traumatic, and he had back pain after the previous procedure. After discussion, he agreed to proceed with spinal anesthesia under ultrasound guidance. After obtaining informed consent, routine monitoring and intravenous access premedication was given for a spinal anesthesia procedure with an ultrasound-guided needle-guidance system, and spinal anesthesia was successful at the first attempt.

We used the SonoSite S nerve (SonoSite, Bothell, WA, USA) ultrasound machine with a low-frequency (2-5 MHz) curvilinear transducer. The ultrasound probe was covered with a sterile sleeve with sterile gel applied on the probe of the Infiniti Plus needle-guidance system (Fig. 1). The ultrasound probe was held in the left hand and the needle in the right hand at the clini-

(c) This is an open-access article distributed under the terms of the Creative Commons Attribution Non-Commercial License (http://creativecommons.org/ licenses/by-nc/4.0/), which permits unrestricted non-commercial use, distribution, and reproduction in any medium, provided the original work is properly cited. 

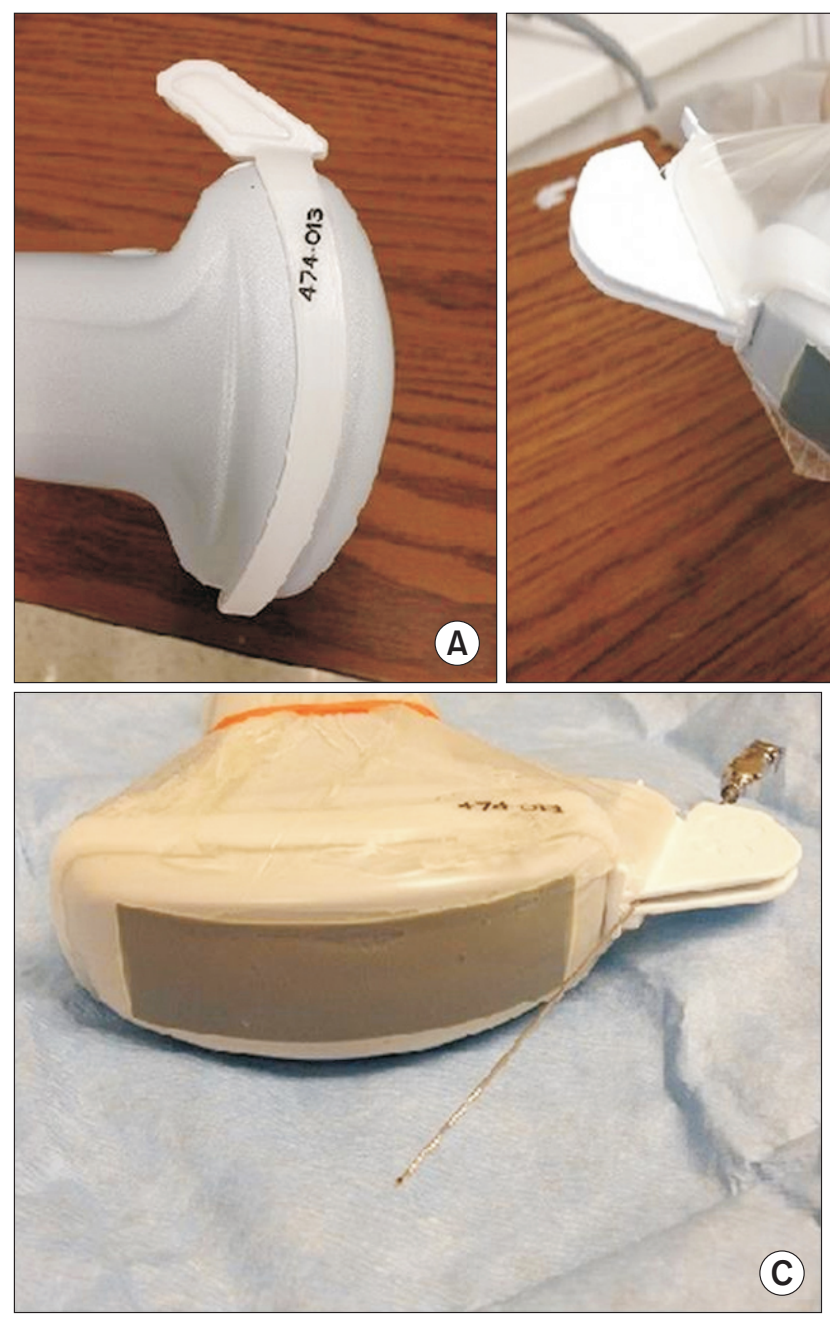

(c)
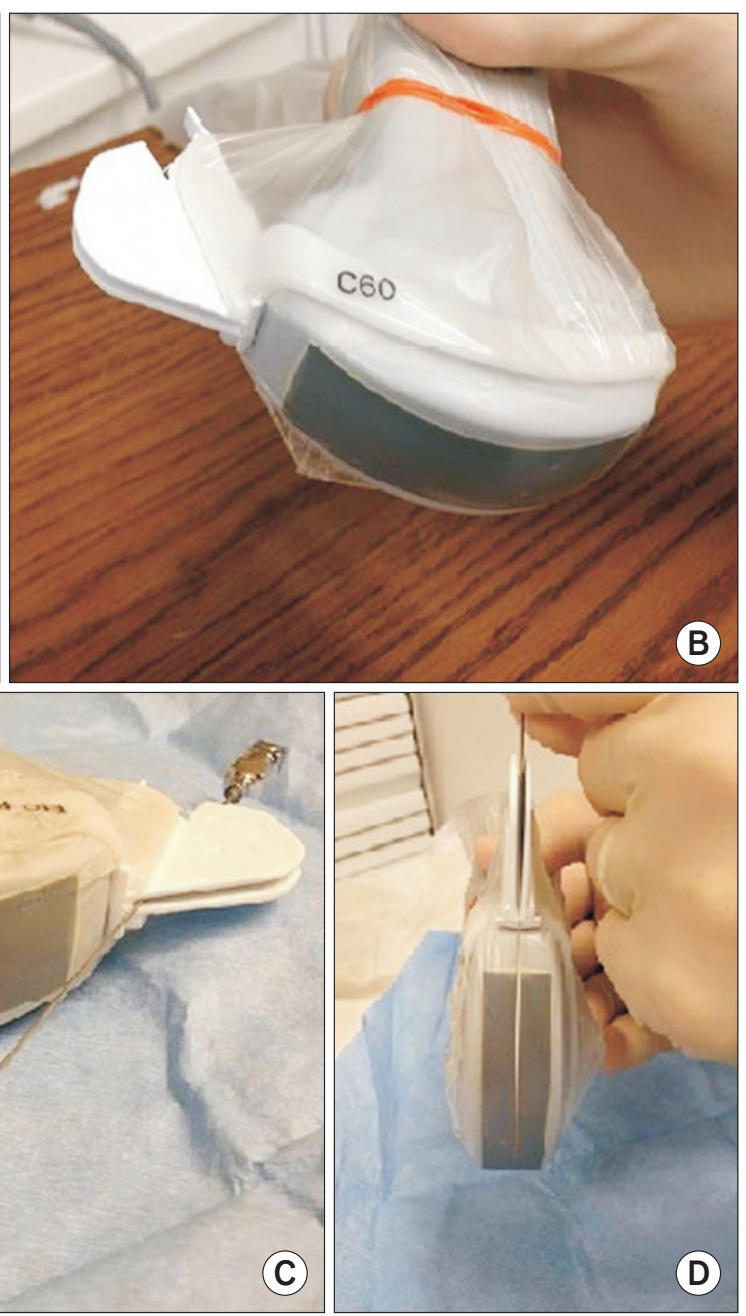

Fig. 1. (A) The non-sterile part of the needle-guidance system was mounted on the ultrasound probe. (B) Gel was applied under a sterile cover and the sterile part of the needle-guidance system was mounted over the sterile cover. (C, D) Echogenic 22 -gauge spinal needle attached to the needle-guidance system. cian's preference, while the ultrasound machine was across the table, in the line of sight of the clinician. Scanning started with the long axis view (paramedian sagittal oblique view) at the sacrum, and then moved cephalad to the lumbar spine to identify the paramedian sagittal oblique interlaminar view at the target lumbar level for the spinal block. We used a paramedian longitudinal oblique approach with needle insertion directed caudal to cephalad. Meticulous care was taken to ensure that the needle insertion site was free of gel. The skin and subcutaneous tissue were infiltrated with $2 \mathrm{ml}$ of $2 \%$ lidocaine.

The intervertebral level used for the attempt was selected based on the relative ease of visualizing the ligamentum flavumdura mater complex. The clinician selected L4-5 in the first case and L3-4 in the second. A 22-gauge, 90-mm-long echogenic spinal needle (Pajunk Medizintechnologie, Geisingen, Germany) was used in both cases. The spinal needle was guided in a caudal-to-cephalic direction via a paramedian oblique interlaminar approach, with the clinician holding both the probe and the needle. The clinician needs to make subtle adjustments in needle trajectory to improve the visualization of the needle while it is inside the needle guidance system. Additionally, the trajectory is changed while the needle is in the muscles to reach the interlaminar space.

When the needle tip reached the interlaminar space close to the posterior complex, the needle visualization deteriorated, while the clinician felt increased resistance. On advancing the needle a total of $4 \mathrm{~cm}$, a sensation of giving was felt on entering the intrathecal space.

After confirming the flow of CSF in both cases, $15 \mathrm{mg}$ bupivacaine was injected. Local anesthetic spread within the subarachnoid space was verified using ultrasound color Doppler images in the transverse interlaminar view.

Each patient required one needle insertion under ultrasound. The anesthesiologist rated the difficulty of insertion as moderate in both patients and both patients reported a very good satisfaction score with no complications. We were able to visualize the needle all the way to the interlaminar space, until the needle approached the posterior complex. 
Both patients underwent successful surgery with spinal anesthesia and were discharged without any complications of spinal anesthesia.

To our knowledge, this is the first report on the use of lowfrequency real-time ultrasound-guided spinal anesthesia with an echogenic spinal needle using needle guidance in clinical prac- tice. We demonstrated that the needle-guidance system is practical, with improved visualization of the needle at depths greater than $4 \mathrm{~cm}$ in patients with a history of difficult spinal anesthesia. Future studies are needed to establish its utility with a larger number of patients and a wider variety of block techniques.

\section{References}

1. Fettes PD, Jansson JR, Wildsmith JA. Failed spinal anaesthesia: mechanisms, management, and prevention. Br J Anaesth 2009; 102: 739-48.

2. Conroy PH, Luyet C, McCartney CJ, McHardy PG. Real-time ultrasound-guided spinal anaesthesia: a prospective observational study of a new approach. Anesthesiol Res Pract 2013; 2013: 525818.

3. Sng BL, Lim Y, Sia AT. An observational prospective cohort study of incidence and characteristics of failed spinal anaesthesia for caesarean section. Int J Obstet Anesth 2009; 18: 237-41.

4. Hebard S, Hocking G, Murray K. Two-dimensional mapping to assess direction and magnitude of needle tip error in ultrasound-guided regional anaesthesia. Anaesth Intensive Care 2011; 39: 1076-81. 